\title{
Numerical solutions for the linear and nonlinear singular boundary value problems using Laguerre wavelets
}

Fengying Zhou and Xiaoyong $\mathrm{Xu}^{*}$

"Correspondence: xxy@ecit.cn School of Science, East China University of Technology,

Nanchang, 330013, P.R. China

\begin{abstract}
In this paper, a collocation method based on Laguerre wavelets is proposed for the numerical solutions of linear and nonlinear singular boundary value problems. Laguerre wavelet expansions together with operational matrix of integration are used to convert the problems into systems of algebraic equations which can be efficiently solved by suitable solvers. Illustrative examples are given to demonstrate the validity and applicability of this technique, and the results have been compared with the exact solutions.
\end{abstract}

MSC: $42 C 40 ; 65 D 30$

Keywords: Laguerre wavelets; collocation method; singular boundary value problems; operational matrix of integration

\section{Introduction}

Singular boundary value problems (BVPs) for ordinary differential equations occur frequently in the fields of engineering and science such as gas dynamics, nuclear physics, atomic structures and chemical reactions [1]. In most cases, we do not always find the exact solutions for the singular boundary values problems via analytical methods. In this case, it is very meaningful to give the high precision numerical solutions for this kind of problem by numerical methods.

The purpose of this paper is to develop a Laguerre wavelets collocation method as an alternative method to solve singular two-point boundary value problems of the form [2]

$$
\mu^{\prime \prime}(x)+\frac{L}{x} \mu^{\prime}(x)+f(x, \mu(x))=g(x), \quad x \in D
$$

subject to the following initial and boundary conditions:

$$
\begin{array}{rll}
\text { Type I : } & \mu(a)=\alpha_{1}, & \mu(b)=\beta_{1}, \\
\text { Type II : } & \mu^{\prime}(a)=\alpha_{2}, & \mu(b)=\beta_{2}, \\
\text { Type III : } & \mu(a)=\alpha_{3}, & \mu^{\prime}(a)=\beta_{3},
\end{array}
$$

(c) 2016 Zhou and $\mathrm{Xu}$. This article is distributed under the terms of the Creative Commons Attribution 4.0 International License (http://creativecommons.org/licenses/by/4.0/), which permits unrestricted use, distribution, and reproduction in any medium, provided you give appropriate credit to the original author(s) and the source, provide a link to the Creative Commons license, and indicate if changes were made. 
and the most general mixed boundary conditions

$$
\text { Type IV : } \quad a_{1} \mu(a)+a_{2} \mu^{\prime}(a)=\alpha_{4}, \quad b_{1} \mu(b)+b_{2} \mu^{\prime}(b)=\beta_{4},
$$

where $L, a_{i}, b_{i}, i=1,2, \alpha_{i}, \beta_{i}, i=1,2,3,4$ are known constants, $D$ is an open or half-open interval with endpoints $a$ and $b, f(x, \mu(x))$ and $g(x)$ are continuous real valued functions on $D$.

Numerous research work has been invested to study the singular BVPs of the form (1)(5). For more details, the reader is kindly recommended to see the survey in [1]. Recently, many researchers have obtained approximations for singular BVPs via various methods. For example, Kanth and Aruna applied He's variational iteration method [3], Chang employed the Taylor series method [4], Singh and Kumar proposed a new technique based on Green's function [5], Sahlan and Hashemizadeh used the wavelet Galerkin method [6], Arqub et al. studied a continuous genetic algorithm [7], Ebaid used the Adomian decomposition method [8], Goh et al. developed a quartic B-spline method [9], and Nasab proposed the Chebyshev finite difference method [10]. Moreover, orthogonal polynomial methods have seen significant achievements in dealing with singular boundary value problems, for example, Legendre polynomials [11], Chebyshev polynomials [2], Bernstein polynomials [12], Laguerre polynomials [13], Bessel polynomials [14], Hermite polynomials [15], and Bernoulli polynomials [16]. Note that these polynomials are supported on the whole interval. This is obviously a defect for certain analysis work, especially problems involving local functions vanishing outside a short interval. However, one advantage of wavelet analysis is the ability to perform a local analysis. This characteristic of time-frequency localization can overcome the defect and allows us to obtain very accurate numerical solutions.

There are two different approaches for solving differential equations. One approach is based on converting differential equations into integral equations through integration, approximating various signals involved in the equation by truncated orthogonal series, and using the operational matrix of integration, to eliminate the integral operations [17]. Another one is based on using operational matrix of derivatives in order to reduce the problem into solving a system of linear or nonlinear algebraic equations. There are some papers in the literature about using the operational matrix of derivatives to solve differential equations $[6,18,19]$.

The rest of this paper is organized as follows. In Section 2, we introduce the Laguerre wavelets and the operational matrix of integration. The error estimation of the Laguerre wavelets expansion is also given. In Section 3, the proposed method is used to approximate solutions of the problems. Section 4 gives several examples to test the proposed method. A conclusion is drawn in Section 5.

\section{Laguerre wavelets and their properties}

\subsection{Wavelets and Laguerre wavelets}

Wavelets constitute a family of functions constructed from dilation and translation of a single function called the mother wavelet. When the dilation parameter $a$ and the translation parameter $b$ vary continuously, we have the following family of continuous wavelets:

$$
\psi_{a, b}(t)=|a|^{-1 / 2} \psi\left(\frac{t-b}{a}\right), \quad a, b \in \mathbb{R}, a \neq 0 .
$$


If we restrict the parameters $a$ and $b$ to discrete values as $a=a_{0}^{-j}, b=m b_{0} a_{0}^{-j}$, where $a_{0}>1$, $b_{0}>0$, and $j, m$ are positive integers, we have the following family of discrete wavelets:

$$
\psi_{j, m}(t)=\left|a_{0}\right|^{j / 2} \psi\left(a_{0}^{j} t-m b_{0}\right),
$$

which form a wavelet basis for $L^{2}(\mathbb{R})$. In particular, when $a_{0}=2$ and $b_{0}=1$, then $\psi_{j, m}(t)$ form an orthonormal basis.

The Laguerre wavelets $\psi_{n, m}(t)=\psi(k, n, m, t)$ have four arguments: $k$ can assume any positive integer, $n=1,2,3, \ldots, 2^{k-1}, m$ is the degree of Laguerre polynomials, and $t$ is the normalized time. They are defined on the interval $[0,1)$ as

$$
\psi_{n, m}(t)= \begin{cases}2^{\frac{k}{2}} L_{m}\left(2^{k} t-2 n+1\right), & \frac{n-1}{2^{k-1}} \leq t<\frac{n}{2^{k-1}}, \\ 0, & \text { otherwise }\end{cases}
$$

where $m=0,1,2, \ldots, M-1$ and $M$ is a fixed positive integer, $L_{m}(t)$ are the Laguerre polynomials of degree $m$ which are orthogonal with respect to the weight function $\omega(t)=e^{-t}$ on the interval $[0, \infty)$ and satisfy the following recursive formula:

$$
L_{0}(t)=1, \quad L_{1}(t)=1-t, \quad L_{m+1}(t)=\frac{(2 m+1-t) L_{m}(t)-m L_{m-1}(t)}{m+1}, \quad m=1,2,3, \ldots
$$

A function $\mu(x) \in L^{2}(\mathbb{R})$ defined over $[0,1)$ may be expanded by Laguerre wavelets as

$$
\mu(x)=\sum_{n=1}^{\infty} \sum_{m=0}^{\infty} c_{n, m} \psi_{n, m}(x) .
$$

If the infinite series in equation (6) is truncated, then it can be written by

$$
\mu(x) \cong \sum_{n=1}^{2^{k-1}} \sum_{m=0}^{M-1} c_{n, m} \psi_{n, m}(x)=\mathbf{C}^{T} \boldsymbol{\Psi}(x),
$$

where $\mathbf{C}$ and $\boldsymbol{\Psi}(x)$ are $2^{k-1} M \times 1$ matrices given by

$$
\begin{aligned}
& \mathbf{C}=\left(\begin{array}{lllllllllllll}
c_{1,0} & c_{1,1} & \cdots & c_{1, M-1} & c_{2,0} & c_{2,1} & \cdots & c_{2, M-1} & \cdots & c_{2^{k-1,0}} & c_{2^{k-1,1}} & \cdots & c_{2^{k-1}, M-1}
\end{array}\right)^{T}, \\
& \boldsymbol{\Psi}(x)=\left(\begin{array}{lllllllllllll}
\psi_{1,0} & \psi_{1,1} & \cdots & \psi_{1, M-1} & \psi_{2,0} & \psi_{2,1} & \cdots & \psi_{2, M-1} & \cdots & \psi_{2^{k-1,0}} & \psi_{2^{k-1,1}} & \cdots & \psi_{2^{k-1, M-1}}
\end{array}\right)^{T} .
\end{aligned}
$$

Since the truncated Laguerre wavelets series can be an approximate solution of singular BVPs, one has an error function $E(x)$ for $\mu(x)$ as follows:

$$
E(x)=\left|\mu(x)-\mathbf{C}^{T} \boldsymbol{\Psi}(x)\right| .
$$

The following theorem gives the error estimation of the Laguerre wavelets expansion.

Theorem 1 Suppose that $\mu(x) \in C^{m}[0,1]$ and $\mathbf{C}^{T} \mathbf{\Psi}(x)$ is the approximate solution using Laguerre wavelets. Then the error bound would be given by

$$
\|E(x)\| \leq\left\|\frac{2}{m ! 4^{m} 2^{m(k-1)}} \max _{x \in[0,1]}\left|\mu^{(m)}(x)\right|\right\| .
$$


Proof We divide the interval $[0,1]$ into $2^{k-1}$ subintervals $I_{n}=\left[\frac{n-1}{2^{k-1}}, \frac{n}{2^{k-1}}\right], n=1,2,3, \ldots, 2^{k-1}$. Then

$$
\begin{aligned}
\|E(x)\|^{2} & =\int_{0}^{1}\left(\mu(x)-\mathbf{C}^{T} \boldsymbol{\Psi}(x)\right)^{2} d x=\sum_{n=1}^{2^{k-1}} \int_{\frac{n-1}{2^{k-1}}}^{\frac{n}{2^{k-1}}}\left(\mu(x)-\mathbf{C}^{T} \boldsymbol{\Psi}(x)\right)^{2} d x \\
& \leq \sum_{n=1}^{2^{k-1}} \int_{\frac{n-1}{2^{k-1}}}^{\frac{n}{2^{k-1}}}\left(\mu(x)-P_{m}(x)\right)^{2} d x,
\end{aligned}
$$

where $P_{m}(x)$ is the interpolating polynomial of degree $m$ which approximates $\mu(x)$ on $I_{n}$. By using the maximum error estimate for the polynomial on $I_{n}$, we have

$$
\begin{aligned}
\|E(x)\|^{2} & \leq \sum_{n=1}^{2^{k-1}} \int_{\frac{n-1}{2^{k-1}}}^{\frac{n}{2^{k-1}}}\left(\frac{2}{m ! 4^{m} 2^{m(k-1)}} \max _{x \in I_{n}}\left|\mu^{(m)}(x)\right|\right)^{2} d x \\
& \leq \sum_{n=1}^{2^{k-1}} \int_{\frac{n-1}{2^{k-1}}}^{\frac{n}{2^{k-1}}}\left(\frac{2}{m ! 4^{m} 2^{m(k-1)}} \max _{x \in[0,1]}\left|\mu^{(m)}(x)\right|\right)^{2} d x \\
& =\int_{0}^{1}\left(\frac{2}{m ! 4^{m} 2^{m(k-1)}} \max _{x \in[0,1]}\left|\mu^{(m)}(x)\right|\right)^{2} d x \\
& =\left\|\frac{2}{m ! 4^{m} 2^{m(k-1)}} \max _{x \in[0,1]}\left|\mu^{(m)}(x)\right|\right\|^{2},
\end{aligned}
$$

where we have used the well-known maximum error bound for the interpolation.

\subsection{Operational matrix of integration (OMI)}

In this section, we give the structure of OMI for Laguerre wavelets with $k=2$ and $M=3$. In this case, the six basis functions are given by

$$
\begin{aligned}
& \psi_{1,0}(t)=2, \\
& \psi_{1,1}(t)=4-8 t, \\
& \psi_{1,2}(t)=7-24 t+16 t^{2},
\end{aligned}
$$

on $\left[0, \frac{1}{2}\right)$ and

$$
\begin{aligned}
& \psi_{2,0}(t)=2, \\
& \psi_{2,1}(t)=8-8 t \\
& \psi_{2,2}(t)=23-40 t+16 t^{2}
\end{aligned}
$$

on $\left[\frac{1}{2}, 1\right)$. Let $\boldsymbol{\Psi}_{6}(t)=\left(\psi_{1,0}(t) \psi_{1,1}(t) \psi_{1,2}(t) \psi_{2,0}(t) \psi_{2,1}(t) \psi_{2,2}(t)\right)^{T}$. By integrating $(8)$ and (9) from 0 to $t$ and representing them in the matrix form, we obtain

$$
\begin{aligned}
& \int_{0}^{t} \psi_{1,0}(s) d s=\left(\begin{array}{cccccc}
\frac{1}{2} & -\frac{1}{4} & 0 & \frac{1}{2} & 0 & 0
\end{array}\right) \Psi_{6}(t), \\
& \int_{0}^{t} \psi_{1,1}(s) d s=\left(\begin{array}{cccccc}
\frac{3}{8} & \frac{1}{4} & -\frac{1}{4} & \frac{1}{2} & 0 & 0
\end{array}\right) \Psi_{6}(t)
\end{aligned}
$$




$$
\begin{aligned}
& \int_{0}^{t} \psi_{1,2}(s) d s=\left(\begin{array}{llllll}
\frac{13}{24} & 0 & \frac{1}{4} & \frac{7}{12} & 0 & 0
\end{array}\right) \boldsymbol{\Psi}_{6}(t)-\frac{1}{4} \psi_{1,3}(t) \\
& \int_{0}^{t} \psi_{2,0}(s) d s=\left(\begin{array}{llllll}
0 & 0 & 0 & \frac{1}{2} & -\frac{1}{4} & 0
\end{array}\right) \boldsymbol{\Psi}_{6}(t) \\
& \int_{0}^{t} \psi_{2,1}(s) d s=\left(\begin{array}{llllll}
0 & 0 & 0 & \frac{3}{8} & \frac{1}{4} & -\frac{1}{4}
\end{array}\right) \boldsymbol{\Psi}_{6}(t) \\
& \int_{0}^{t} \psi_{2,2}(s) d s=\left(\begin{array}{llllll}
0 & 0 & 0 & \frac{13}{24} & 0 & \left.\frac{1}{4}\right) \boldsymbol{\Psi}_{6}(t)-\frac{1}{4} \psi_{2,3}(t) .
\end{array}\right.
\end{aligned}
$$

Thus

$$
\int_{0}^{t} \boldsymbol{\Psi}_{6}(s) d s=\mathbf{P}_{6 \times 6} \boldsymbol{\Psi}_{6}(t)+\widetilde{\boldsymbol{\Psi}}_{6}(t)
$$

where

$$
\mathbf{P}_{6 \times 6}=\left(\begin{array}{cccccc}
\frac{1}{2} & -\frac{1}{4} & 0 & \frac{1}{2} & 0 & 0 \\
\frac{3}{8} & \frac{1}{4} & -\frac{1}{4} & \frac{1}{2} & 0 & 0 \\
\frac{13}{24} & 0 & \frac{1}{4} & \frac{7}{12} & 0 & 0 \\
0 & 0 & 0 & \frac{1}{2} & -\frac{1}{4} & 0 \\
0 & 0 & 0 & \frac{3}{8} & \frac{1}{4} & -\frac{1}{4} \\
0 & 0 & 0 & \frac{13}{24} & 0 & \frac{1}{4}
\end{array}\right)
$$

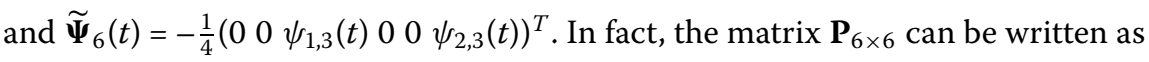

$$
\mathbf{P}_{6 \times 6}=\left(\begin{array}{ll}
\mathbf{A}_{3 \times 3} & \mathbf{B}_{3 \times 3} \\
\mathbf{0}_{3 \times 3} & \mathbf{A}_{3 \times 3}
\end{array}\right),
$$

where

$$
\mathbf{A}_{3 \times 3}=\left(\begin{array}{ccc}
\frac{1}{2} & -\frac{1}{4} & 0 \\
\frac{3}{8} & \frac{1}{4} & -\frac{1}{4} \\
\frac{13}{24} & 0 & \frac{1}{4}
\end{array}\right) \quad \text { and } \quad \mathbf{B}_{3 \times 3}=\left(\begin{array}{ccc}
\frac{1}{2} & 0 & 0 \\
\frac{1}{2} & 0 & 0 \\
\frac{7}{12} & 0 & 0
\end{array}\right)
$$

Unfortunately, for general $k$ and $M$, operational matrix of integration does not have a regular expression. So when dealing with the problems, we need to pre-calculate the corresponding operational matrix of integration $\mathbf{P}$ and $\widetilde{\boldsymbol{\Psi}}(t)$ for different $k$ and $M$ such that

$$
\int_{0}^{t} \boldsymbol{\Psi}(s) d s=\mathbf{P} \boldsymbol{\Psi}(t)+\widetilde{\boldsymbol{\Psi}}(t)
$$

For example, for $k=3$ and $M=3$, the operational matrix of integration is given by

$$
\mathbf{P}=\left(\begin{array}{cccc}
\mathbf{A} & \mathbf{B} & \mathbf{B} & \mathbf{B} \\
\mathbf{0} & \mathbf{A} & \mathbf{B} & \mathbf{B} \\
\mathbf{0} & \mathbf{0} & \mathrm{A} & \mathbf{B} \\
\mathbf{0} & \mathbf{0} & \mathbf{0} & \mathbf{A}
\end{array}\right)
$$


where $\mathbf{A}$ and $\mathbf{B}$ are $3 \times 3$ matrices given by

$$
\mathbf{A}=\left(\begin{array}{ccc}
\frac{1}{4} & -\frac{1}{8} & 0 \\
\frac{3}{16} & \frac{1}{8} & -\frac{1}{8} \\
\frac{13}{48} & 0 & \frac{1}{8}
\end{array}\right), \quad \mathbf{B}=\left(\begin{array}{ccc}
\frac{1}{4} & 0 & 0 \\
\frac{1}{4} & 0 & 0 \\
\frac{7}{24} & 0 & 0
\end{array}\right)
$$

and

$$
\widetilde{\boldsymbol{\Psi}}(t)=-\frac{1}{8}\left(\begin{array}{llllllllllll}
0 & 0 & \psi_{1,3}(t) & 0 & 0 & \psi_{2,3}(t) & 0 & 0 & \psi_{3,3}(t) & 0 & 0 & \left.\psi_{4,3}(t)\right)^{T}
\end{array}\right.
$$

\section{Description of the proposed method}

In this section, we will use Laguerre wavelets operational matrix of integration combining collocation method to solve linear or nonlinear singular boundary value problems. Assume that

$$
\mu^{\prime \prime}(x)=\mathbf{C}^{T} \boldsymbol{\Psi}(x)
$$

where $\mathbf{C}$ is an unknown vector which should be determined and $\boldsymbol{\Psi}(x)$ is the vector defined in (7). Equation (11) is integrated two times with respect to $x$. In this way, the solution $\mu(x)$ and its two derivatives are expressed in terms of Laguerre wavelets functions and their integrals. Consider the collocation points

$$
x_{i}=\frac{2 i-1}{2^{k} M}, \quad i=1,2,3, \ldots, 2^{k-1} M .
$$

The expressions of $\mu(x), \mu^{\prime}(x)$, and $\mu^{\prime \prime}(x)$ are substituted in the given differential equations and discretization is applied using the collocation points. Thus we get a system of equations with $2^{k-1} M$ unknowns

$$
\mu^{\prime \prime}\left(x_{i}\right)+\frac{L}{x_{i}} \mu^{\prime}\left(x_{i}\right)+f\left(x_{i}, \mu\left(x_{i}\right)\right)=g\left(x_{i}\right), \quad i=1,2,3, \ldots, 2^{k-1} M
$$

Then we can obtain the unknown vector $\mathbf{C}$ by solving this system through the well-known Newton iterative method with the aid of Matlab. The approximate solution can easily be recovered by inserting $\mathbf{C}$ into the corresponding expression of $\mu(x)$. We further explain the method with the help of specific boundary conditions. In this paper, we consider the four different types of boundary conditions (2)-(4) and derive the expressions of $\mu(x)$, $\mu^{\prime}(x)$, and $\mu^{\prime \prime}(x)$, respectively. For simplicity, we take $a=0$ and $b=1$. Before the further description of the proposed method, we introduce the following notations first:

$$
\boldsymbol{\Psi}(1):=\lim _{x \rightarrow 1^{-}} \boldsymbol{\Psi}(x), \quad \int_{0}^{1} \widetilde{\boldsymbol{\Psi}}(s) d s:=\lim _{x \rightarrow 1^{-}} \int_{0}^{x} \widetilde{\boldsymbol{\Psi}}(s) d s, \quad \text { and } \quad \widetilde{\boldsymbol{\Psi}}(1):=\lim _{x \rightarrow 1^{-}} \widetilde{\boldsymbol{\Psi}}(x) .
$$

Type I. Consider boundary conditions (2). Assume

$$
\mu^{\prime \prime}(x)=\mathbf{C}^{T} \boldsymbol{\Psi}(x)
$$

By integrating (13) two times with respect to $x$ and together with relation (10), we obtain

$$
\mu^{\prime}(x)=\mu^{\prime}(0)+\mathbf{C}^{T}(\mathbf{P} \boldsymbol{\Psi}(x)+\widetilde{\boldsymbol{\Psi}}(x))
$$




$$
\mu(x)=\alpha_{1}+\mu^{\prime}(0) x+\mathbf{C}^{T}\left(\mathbf{P}^{2} \mathbf{\Psi}(x)+\mathbf{P} \widetilde{\mathbf{\Psi}}(x)+\int_{0}^{x} \widetilde{\Psi}(s) d s\right) .
$$

Putting $x=1$ in (14) gives

$$
\mu^{\prime}(0)=\beta_{1}-\alpha_{1}-\mathbf{C}^{T}\left(\mathbf{P}^{2} \boldsymbol{\Psi}(1)+\mathbf{P} \widetilde{\Psi}(1)+\int_{0}^{1} \widetilde{\Psi}(s) d s\right) .
$$

So

$$
\begin{aligned}
\mu(x)= & \alpha_{1}+\left(\beta_{1}-\alpha_{1}-\mathbf{C}^{T}\left(\mathbf{P}^{2} \boldsymbol{\Psi}(1)+\mathbf{P} \widetilde{\boldsymbol{\Psi}}(1)+\int_{0}^{1} \widetilde{\mathbf{\Psi}}(s) d s\right)\right) x \\
& +\mathbf{C}^{T}\left(\mathbf{P}^{2} \boldsymbol{\Psi}(x)+\mathbf{P} \widetilde{\boldsymbol{\Psi}}(x)+\int_{0}^{x} \widetilde{\mathbf{\Psi}}(s) d s\right) .
\end{aligned}
$$

Type II. Consider boundary conditions (3). Assume

$$
\mu^{\prime \prime}(x)=\mathbf{C}^{T} \boldsymbol{\Psi}(x)
$$

By integrating (15) two times with respect to $x$ and by equation (10), we get

$$
\begin{aligned}
& \mu^{\prime}(x)=\alpha_{2}+\mathbf{C}^{T}(\mathbf{P} \boldsymbol{\Psi}(x)+\widetilde{\mathbf{\Psi}}(x)), \\
& \mu(x)=\mu(0)+\mu^{\prime}(0) x+\mathbf{C}^{T}\left(\mathbf{P}^{2} \boldsymbol{\Psi}(x)+\mathbf{P} \widetilde{\boldsymbol{\Psi}}(x)+\int_{0}^{x} \widetilde{\boldsymbol{\Psi}}(s) d s\right) .
\end{aligned}
$$

Putting $x=1$ in (16), it follows that

$$
\mu(0)=\beta_{2}-\alpha_{2}-\mathbf{C}^{T}\left(\mathbf{P}^{2} \boldsymbol{\Psi}(1)+\mathbf{P} \widetilde{\boldsymbol{\Psi}}(1)+\int_{0}^{1} \widetilde{\boldsymbol{\Psi}}(s) d s\right) .
$$

Thus

$$
\begin{aligned}
\mu(x)= & \beta_{2}-\alpha_{2}-\mathbf{C}^{T}\left(\mathbf{P}^{2} \boldsymbol{\Psi}(1)+\mathbf{P} \widetilde{\boldsymbol{\Psi}}(1)+\int_{0}^{1} \widetilde{\boldsymbol{\Psi}}(s) d s\right)+\alpha_{2} x \\
& +\mathbf{C}^{T}\left(\mathbf{P}^{2} \boldsymbol{\Psi}(x)+\mathbf{P} \widetilde{\boldsymbol{\Psi}}(x)+\int_{0}^{x} \widetilde{\boldsymbol{\Psi}}(s) d s\right) .
\end{aligned}
$$

Type III. Consider boundary conditions (4). Assume

$$
\mu^{\prime \prime}(x)=\mathbf{C}^{T} \boldsymbol{\Psi}(x) .
$$

By integrating (17) two times with respect to $x$ and by relation (10), we obtain

$$
\begin{aligned}
& \mu^{\prime}(x)=\beta_{3}+\mathbf{C}^{T}(\mathbf{P} \boldsymbol{\Psi}(x)+\widetilde{\Psi}(x)), \\
& \mu(x)=\alpha_{3}+\beta_{3} x+\mathbf{C}^{T}\left(\mathbf{P}^{2} \boldsymbol{\Psi}(x)+\mathbf{P} \widetilde{\Psi}(x)+\int_{0}^{x} \widetilde{\Psi}(s) d s\right) .
\end{aligned}
$$

Type IV. Consider boundary conditions (5). Assume

$$
\mu^{\prime \prime}(x)=\mathbf{C}^{T} \boldsymbol{\Psi}(x) .
$$


By integrating (19) two times with respect to $x$ and by relation (10), we have

$$
\begin{aligned}
& \mu^{\prime}(x)=\mu^{\prime}(0)+\mathbf{C}^{T}(\mathbf{P} \boldsymbol{\Psi}(x)+\widetilde{\boldsymbol{\Psi}}(x)) \\
& \mu(x)=\mu(0)+\mu^{\prime}(0) x+\mathbf{C}^{T}\left(\mathbf{P}^{2} \boldsymbol{\Psi}(x)+\mathbf{P} \widetilde{\boldsymbol{\Psi}}(x)+\int_{0}^{x} \widetilde{\boldsymbol{\Psi}}(s) d s\right) .
\end{aligned}
$$

Putting $x=1$ in (20) and (21) leads to

$$
\begin{aligned}
& \mu^{\prime}(1)=\mu^{\prime}(0)+\mathbf{C}^{T}(\mathbf{P} \boldsymbol{\Psi}(1)+\widetilde{\boldsymbol{\Psi}}(1)) \\
& \mu^{\prime}(0)=\mu(1)-\mu(0)-\mathbf{C}^{T}\left(\mathbf{P}^{2} \boldsymbol{\Psi}(1)+\mathbf{P} \widetilde{\boldsymbol{\Psi}}(1)+\int_{0}^{1} \widetilde{\boldsymbol{\Psi}}(s) d s\right) .
\end{aligned}
$$

Hence equations in (5) turn into the following forms:

$$
\begin{aligned}
& a_{1} \mu(0)+a_{2}\left(\mu(1)-\mu(0)-\mathbf{C}^{T}\left(\mathbf{P}^{2} \boldsymbol{\Psi}(1)+\mathbf{P} \widetilde{\boldsymbol{\Psi}}(1)+\int_{0}^{1} \widetilde{\boldsymbol{\Psi}}(s) d s\right)\right)=\alpha_{4}, \\
& b_{1} \mu(1)+b_{2}\left(\mu(1)-\mu(0)-\mathbf{C}^{T}\left(\mathbf{P}^{2} \boldsymbol{\Psi}(1)+\mathbf{P} \widetilde{\boldsymbol{\Psi}}(1)+\int_{0}^{1} \widetilde{\boldsymbol{\Psi}}(s) d s\right)\right. \\
& \left.+\mathbf{C}^{T}(\mathbf{P} \boldsymbol{\Psi}(1)+\widetilde{\boldsymbol{\Psi}}(1))\right)=\beta_{4} .
\end{aligned}
$$

Observe that we consider $\mu(0)$ and $\mu(1)$ as unknown variables in equations (23) and (24). Equations (12) together with (23) and (24) generate $2^{k-1} M+2$ equations, which can be solved by using Newton's iterative method.

\section{Numerical examples}

In order to demonstrate the efficiency and applicability of the proposed method, several linear or nonlinear singular two-point BVPs are studied. We also compare the approximate solution with the exact solution. All computations are performed by Matlab.

Example 1 Consider the following linear singular two-point BVP:

$$
\mu^{\prime \prime}(x)+\frac{1}{x} \mu^{\prime}(x)+\frac{1}{1-x} \mu(x)=4 \cos x-5 x \sin x+\frac{x^{3}}{1-x} \cos x, \quad 0<x<1,
$$

subject to the boundary conditions

$$
\mu(0)=1, \quad \mu(1)=\cos 1 .
$$

The exact solution is given by $\mu(x)=x^{2} \cos x$. We solve this equation by Laguerre wavelets collocation method with $k=2,3$ and $M=3,4,5,6,7$. Tables 1 and 2 show the comparison between the absolute error of exact and approximate solutions for various values of $M$ with $k=2,3$. As can be seen in the tables, the Laguerre wavelet solution is very close to the exact one.

Example 2 Consider the following nonlinear Lane-Emden equation [20]:

$$
\mu^{\prime \prime}(x)+\frac{6}{x} \mu^{\prime}(x)+14 \mu(x)+4 \mu(x) \ln (\mu(x))=0, \quad 0<x<1,
$$


Table 1 Comparison of absolute errors for Example $1(k=2)$

\begin{tabular}{lllllll}
\hline $\boldsymbol{x}$ & Exact solution & $\begin{array}{l}\text { Absolute error } \\
\boldsymbol{M}=\mathbf{3}\end{array}$ & $\begin{array}{l}\text { Absolute error } \\
\boldsymbol{M = 4}\end{array}$ & $\begin{array}{l}\text { Absolute error } \\
\boldsymbol{M}=\mathbf{5}\end{array}$ & $\begin{array}{l}\text { Absolute error } \\
\boldsymbol{M}=\mathbf{6}\end{array}$ & $\begin{array}{l}\text { Absolute error } \\
\boldsymbol{M = 7}\end{array}$ \\
\hline 0.1 & 0.00995004165278026 & $5.05015 \mathrm{e}-5$ & $1.15174 \mathrm{e}-5$ & $1.67248 \mathrm{e}-7$ & $1.94092 \mathrm{e}-8$ & $2.04877 \mathrm{e}-10$ \\
0.2 & 0.0392026631136497 & $6.99671 \mathrm{e}-5$ & $1.49598 \mathrm{e}-5$ & $2.08568 \mathrm{e}-7$ & $2.37033 \mathrm{e}-8$ & $2.44321 \mathrm{e}-10$ \\
0.3 & 0.0859802840213046 & $8.61420 \mathrm{e}-5$ & $1.65555 \mathrm{e}-5$ & $2.27808 \mathrm{e}-7$ & $2.59825 \mathrm{e}-8$ & $2.64413 \mathrm{e}-10$ \\
0.4 & 0.147369759040462 & $8.52543 \mathrm{e}-5$ & $1.74995 \mathrm{e}-5$ & $2.42605 \mathrm{e}-7$ & $2.71268 \mathrm{e}-8$ & $2.74407 \mathrm{e}-10$ \\
0.5 & 0.219395640472593 & $8.52773 \mathrm{e}-5$ & $1.84611 \mathrm{e}-5$ & $2.42878 \mathrm{e}-7$ & $2.80195 \mathrm{e}-8$ & $2.76631 \mathrm{e}-10$ \\
0.6 & 0.297120821367484 & $5.87600 \mathrm{e}-5$ & $1.39445 \mathrm{e}-5$ & $1.76695 \mathrm{e}-7$ & $2.15210 \mathrm{e}-8$ & $2.10918 \mathrm{e}-10$ \\
0.7 & 0.374772671769399 & $3.90650 \mathrm{e}-5$ & $1.02566 \mathrm{e}-5$ & $1.41817 \mathrm{e}-7$ & $1.58798 \mathrm{e}-8$ & $1.56994 \mathrm{e}-10$ \\
0.8 & 0.445892293982186 & $4.85804 \mathrm{e}-5$ & $6.65266 \mathrm{e}-6$ & $9.25693 \mathrm{e}-8$ & $1.04099 \mathrm{e}-8$ & $1.06686 \mathrm{e}-10$ \\
0.9 & 0.503504074299238 & $2.34107 \mathrm{e}-5$ & $3.09612 \mathrm{e}-6$ & $5.98109 \mathrm{e}-8$ & $4.99262 \mathrm{e}-9$ & $5.71941 \mathrm{e}-11$ \\
\hline
\end{tabular}

Table 2 Comparison of absolute errors for Example $1(k=3)$

\begin{tabular}{lllllll}
\hline $\boldsymbol{x}$ & Exact solution & $\begin{array}{l}\text { Absolute error } \\
\mathbf{M = 3}\end{array}$ & $\begin{array}{l}\text { Absolute error } \\
\mathbf{M = 4}\end{array}$ & $\begin{array}{l}\text { Absolute error } \\
\mathbf{M = 5}\end{array}$ & $\begin{array}{l}\text { Absolute error } \\
\boldsymbol{M = 6}\end{array}$ & $\begin{array}{l}\text { Absolute error } \\
\boldsymbol{M = 7}\end{array}$ \\
\hline 0.1 & 0.00995004165278026 & $6.09766 \mathrm{e}-6$ & $1.01327 \mathrm{e}-6$ & $4.46956 \mathrm{e}-9$ & $3.95315 \mathrm{e}-10$ & $5.99699 \mathrm{e}-13$ \\
0.2 & 0.0392026631136497 & $7.11910 \mathrm{e}-6$ & $1.19433 \mathrm{e}-6$ & $5.22659 \mathrm{e}-9$ & $4.73386 \mathrm{e}-10$ & $5.69475 \mathrm{e}-13$ \\
0.3 & 0.0859802840213046 & $6.96418 \mathrm{e}-6$ & $1.22938 \mathrm{e}-6$ & $5.14196 \mathrm{e}-9$ & $5.02232 \mathrm{e}-10$ & $4.70207 \mathrm{e}-13$ \\
0.4 & 0.147369759040462 & $6.72907 \mathrm{e}-6$ & $1.21170 \mathrm{e}-6$ & $4.73782 \mathrm{e}-9$ & $5.05799 \mathrm{e}-10$ & $3.83082 \mathrm{e}-13$ \\
0.5 & 0.219395640472593 & $5.82617 \mathrm{e}-6$ & $1.18534 \mathrm{e}-6$ & $4.30528 \mathrm{e}-9$ & $4.92414 \mathrm{e}-10$ & $3.16524 \mathrm{e}-13$ \\
0.6 & 0.297120821367484 & $4.41211 \mathrm{e}-6$ & $9.85413 \mathrm{e}-7$ & $3.52994 \mathrm{e}-9$ & $4.11916 \mathrm{e}-10$ & $3.36841 \mathrm{e}-13$ \\
0.7 & 0.374772671769399 & $3.86910 \mathrm{e}-6$ & $7.96955 \mathrm{e}-7$ & $2.86759 \mathrm{e}-9$ & $3.31169 \mathrm{e}-10$ & $3.66096 \mathrm{e}-13$ \\
0.8 & 0.445892293982186 & $2.27080 \mathrm{e}-6$ & $5.61629 \mathrm{e}-7$ & $1.81250 \mathrm{e}-9$ & $2.28235 \mathrm{e}-10$ & $3.48554 \mathrm{e}-13$ \\
0.9 & 0.503504074299238 & $1.73623 \mathrm{e}-6$ & $2.78002 \mathrm{e}-7$ & $9.55841 \mathrm{e}-10$ & $1.18077 \mathrm{e}-10$ & $1.97952 \mathrm{e}-13$ \\
\hline
\end{tabular}

Table 3 Comparison of absolute errors for Example $2(k=2)$

\begin{tabular}{lllllll}
\hline $\boldsymbol{X}$ & Exact solution & $\begin{array}{l}\text { Absolute error } \\
\boldsymbol{M = 3}\end{array}$ & $\begin{array}{l}\text { Absolute error } \\
\boldsymbol{M = 4}\end{array}$ & $\begin{array}{l}\text { Absolute error } \\
\boldsymbol{M = 5}\end{array}$ & $\begin{array}{l}\text { Absolute error } \\
\boldsymbol{M = 6}\end{array}$ & $\begin{array}{l}\text { Absolute error } \\
\boldsymbol{M = 7}\end{array}$ \\
\hline 0.1 & 0.990049833749168 & $4.30380 \mathrm{e}-4$ & $1.19711 \mathrm{e}-5$ & $4.49285 \mathrm{e}-6$ & $3.92356 \mathrm{e}-8$ & $3.85970 \mathrm{e}-8$ \\
0.2 & 0.960789439152323 & $2.87642 \mathrm{e}-4$ & $9.28739 \mathrm{e}-6$ & $3.38741 \mathrm{e}-6$ & $3.57194 \mathrm{e}-8$ & $3.70852 \mathrm{e}-8$ \\
0.3 & 0.913931185271228 & $1.96900 \mathrm{e}-4$ & $8.49488 \mathrm{e}-6$ & $3.48051 \mathrm{e}-6$ & $3.46026 \mathrm{e}-8$ & $3.42054 \mathrm{e}-8$ \\
0.4 & 0.852143788966211 & $2.44586 \mathrm{e}-4$ & $8.14368 \mathrm{e}-6$ & $2.98133 \mathrm{e}-6$ & $2.87292 \mathrm{e}-8$ & $3.09439 \mathrm{e}-8$ \\
0.5 & 0.778800783071405 & $2.29986 \mathrm{e}-4$ & $7.24332 \mathrm{e}-6$ & $2.86993 \mathrm{e}-6$ & $2.88864 \mathrm{e}-8$ & $2.85315 \mathrm{e}-8$ \\
0.6 & 0.697676326071031 & $1.02809 \mathrm{e}-4$ & $6.50098 \mathrm{e}-6$ & $1.33540 \mathrm{e}-6$ & $2.75676 \mathrm{e}-8$ & $1.36518 \mathrm{e}-8$ \\
0.7 & 0.612626394184416 & $5.66525 \mathrm{e}-5$ & $3.52342 \mathrm{e}-6$ & $7.04795 \mathrm{e}-7$ & $1.58048 \mathrm{e}-8$ & $6.79409 \mathrm{e}-9$ \\
0.8 & 0.527292424043049 & $5.30123 \mathrm{e}-5$ & $2.77371 \mathrm{e}-6$ & $3.32497 \mathrm{e}-7$ & $9.96594 \mathrm{e}-9$ & $3.16979 \mathrm{e}-9$ \\
0.9 & 0.444858066222941 & $1.55830 \mathrm{e}-5$ & $1.84094 \mathrm{e}-6$ & $1.50285 \mathrm{e}-7$ & $1.09891 \mathrm{e}-8$ & $1.10573 \mathrm{e}-9$ \\
\hline
\end{tabular}

subject to the boundary conditions

$$
\mu(0)=1, \quad \mu(1)=e^{-1} .
$$

The exact solution is $\mu(x)=e^{-x^{2}}$. We solve this equation by the proposed method with $k=2,3$ and $M=3,4,5,6,7$. Tables 3 and 4 show the comparison between the absolute error of exact and approximate solutions for various values of $M$ with $k=2,3$.

Example 3 Consider the singular initial value problem

$$
\mu^{\prime \prime}(x)+\frac{1}{x} \mu^{\prime}(x)-\mu^{3}(x)+3 \mu^{5}(x)=0, \quad 0<x<1,
$$


Table 4 Comparison of absolute errors for Example $2(k=3)$

\begin{tabular}{lllllll}
\hline $\boldsymbol{x}$ & Exact solution & $\begin{array}{l}\text { Absolute error } \\
\boldsymbol{M = 3}\end{array}$ & $\begin{array}{l}\text { Absolute error } \\
\mathbf{M = 4}\end{array}$ & $\begin{array}{l}\text { Absolute error } \\
\boldsymbol{M = 5}\end{array}$ & $\begin{array}{l}\text { Absolute error } \\
\boldsymbol{M = 6}\end{array}$ & $\begin{array}{l}\text { Absolute error } \\
\boldsymbol{M = ~}\end{array}$ \\
\hline 0.1 & 0.990049833749168 & $2.08605 \mathrm{e}-4$ & $5.07778 \mathrm{e}-7$ & $4.15842 \mathrm{e}-7$ & $2.78811 \mathrm{e}-9$ & $2.71666 \mathrm{e}-9$ \\
0.2 & 0.960789439152323 & $2.21586 \mathrm{e}-4$ & $5.87780 \mathrm{e}-7$ & $4.10797 \mathrm{e}-7$ & $2.80960 \mathrm{e}-9$ & $2.51711 \mathrm{e}-9$ \\
0.3 & 0.913931185271228 & $5.48197 \mathrm{e}-5$ & $1.20537 \mathrm{e}-6$ & $1.38717 \mathrm{e}-7$ & $4.74755 \mathrm{e}-9$ & $9.86147 \mathrm{e}-10$ \\
0.4 & 0.852143788966211 & $1.92271 \mathrm{e}-5$ & $1.45560 \mathrm{e}-6$ & $2.03389 \mathrm{e}-8$ & $5.53697 \mathrm{e}-9$ & $1.07901 \mathrm{e}-10$ \\
0.5 & 0.778800783071405 & $3.85323 \mathrm{e}-5$ & $1.36715 \mathrm{e}-6$ & $5.24896 \mathrm{e}-8$ & $5.19276 \mathrm{e}-9$ & $8.98013 \mathrm{e}-11$ \\
0.6 & 0.697676326071031 & $2.41881 \mathrm{e}-5$ & $1.19571 \mathrm{e}-6$ & $4.21942 \mathrm{e}-8$ & $4.19259 \mathrm{e}-9$ & $4.05933 \mathrm{e}-11$ \\
0.7 & 0.612626394184416 & $1.59949 \mathrm{e}-5$ & $1.01058 \mathrm{e}-6$ & $3.36762 \mathrm{e}-8$ & $3.38714 \mathrm{e}-9$ & $1.56910 \mathrm{e}-11$ \\
0.8 & 0.527292424043049 & $9.22128 \mathrm{e}-6$ & $5.95503 \mathrm{e}-7$ & $2.06618 \mathrm{e}-8$ & $2.03107 \mathrm{e}-9$ & $3.88289 \mathrm{e}-11$ \\
0.9 & 0.444858066222941 & $3.14709 \mathrm{e}-6$ & $2.04191 \mathrm{e}-7$ & $7.61645 \mathrm{e}-9$ & $7.32635 \mathrm{e}-10$ & $6.69331 \mathrm{e}-11$ \\
\hline
\end{tabular}

Table 5 Comparison of absolute errors for Example $3(k=2)$

\begin{tabular}{lllllll}
\hline $\boldsymbol{x}$ & Exact solution & $\begin{array}{l}\text { Absolute error } \\
\mathbf{M = 3}\end{array}$ & $\begin{array}{l}\text { Absolute error } \\
\mathbf{M = 4}\end{array}$ & $\begin{array}{l}\text { Absolute error } \\
\mathbf{M = 5}\end{array}$ & $\begin{array}{l}\text { Absolute error } \\
\mathbf{M = 6}\end{array}$ & $\begin{array}{l}\text { Absolute error } \\
\boldsymbol{M = 7}\end{array}$ \\
\hline 0.1 & 0.995037190209989 & $4.77401 \mathrm{e}-5$ & $2.80938 \mathrm{e}-6$ & $1.19035 \mathrm{e}-6$ & $2.35692 \mathrm{e}-8$ & $2.20068 \mathrm{e}-8$ \\
0.2 & 0.98058067569092 & $3.48537 \mathrm{e}-5$ & $2.03669 \mathrm{e}-6$ & $4.07247 \mathrm{e}-7$ & $2.33623 \mathrm{e}-9$ & $1.54801 \mathrm{e}-8$ \\
0.3 & 0.957826285221151 & $2.38207 \mathrm{e}-5$ & $1.20579 \mathrm{e}-6$ & $3.72277 \mathrm{e}-7$ & $7.17380 \mathrm{e}-9$ & $1.02347 \mathrm{e}-8$ \\
0.4 & 0.928476690885259 & $5.21145 \mathrm{e}-6$ & $8.77799 \mathrm{e}-7$ & $1.37710 \mathrm{e}-7$ & $3.12745 \mathrm{e}-9$ & $5.36581 \mathrm{e}-9$ \\
0.5 & 0.894427190999916 & $7.76968 \mathrm{e}-6$ & $5.82907 \mathrm{e}-7$ & $2.11945 \mathrm{e}-7$ & $3.50210 \mathrm{e}-8$ & $6.35488 \mathrm{e}-9$ \\
0.6 & 0.857492925712544 & $1.12585 \mathrm{e}-4$ & $9.24255 \mathrm{e}-6$ & $4.09098 \mathrm{e}-6$ & $3.15261 \mathrm{e}-7$ & $7.61925 \mathrm{e}-8$ \\
0.7 & 0.81923192051904 & $2.06616 \mathrm{e}-4$ & $1.61932 \mathrm{e}-5$ & $7.30904 \mathrm{e}-6$ & $6.05365 \mathrm{e}-7$ & $1.41539 \mathrm{e}-7$ \\
0.8 & 0.78086880944303 & $2.73469 \mathrm{e}-4$ & $2.16091 \mathrm{e}-5$ & $9.89317 \mathrm{e}-6$ & $8.27822 \mathrm{e}-7$ & $1.92037 \mathrm{e}-7$ \\
0.9 & 0.743294146247166 & $3.30810 \mathrm{e}-4$ & $2.58050 \mathrm{e}-5$ & $1.17167 \mathrm{e}-5$ & $9.91475 \mathrm{e}-7$ & $2.29895 \mathrm{e}-7$ \\
\hline
\end{tabular}

Table 6 Comparison of absolute errors for Example $3(k=3)$

\begin{tabular}{lllllll}
\hline $\boldsymbol{x}$ & Exact solution & $\begin{array}{l}\text { Absolute error } \\
\boldsymbol{M = 3}\end{array}$ & $\begin{array}{l}\text { Absolute error } \\
\mathbf{M = 4}\end{array}$ & $\begin{array}{l}\text { Absolute error } \\
\mathbf{M = 5}\end{array}$ & $\begin{array}{l}\text { Absolute error } \\
\boldsymbol{M = 6}\end{array}$ & $\begin{array}{l}\text { Absolute error } \\
\boldsymbol{M = 7}\end{array}$ \\
\hline 0.1 & 0.995037190209989 & $7.56440 \mathrm{e}-7$ & $5.71715 \mathrm{e}-8$ & $2.72876 \mathrm{e}-9$ & $3.20637 \mathrm{e}-10$ & $3.77395 \mathrm{e}-11$ \\
0.2 & 0.98058067569092 & $7.76980 \mathrm{e}-8$ & $2.63566 \mathrm{e}-8$ & $3.70661 \mathrm{e}-10$ & $2.55319 \mathrm{e}-10$ & $1.92960 \mathrm{e}-11$ \\
0.3 & 0.957826285221151 & $6.00860 \mathrm{e}-8$ & $2.26763 \mathrm{e}-8$ & $1.66112 \mathrm{e}-8$ & $6.70535 \mathrm{e}-10$ & $3.12874 \mathrm{e}-10$ \\
0.4 & 0.928476690885259 & $2.29220 \mathrm{e}-6$ & $1.91073 \mathrm{e}-7$ & $4.98419 \mathrm{e}-8$ & $1.35278 \mathrm{e}-9$ & $8.25356 \mathrm{e}-10$ \\
0.5 & 0.894427190999916 & $2.52812 \mathrm{e}-6$ & $3.30368 \mathrm{e}-7$ & $7.10777 \mathrm{e}-8$ & $1.72866 \mathrm{e}-9$ & $1.16419 \mathrm{e}-9$ \\
0.6 & 0.857492925712544 & $6.98583 \mathrm{e}-6$ & $1.99788 \mathrm{e}-7$ & $1.14244 \mathrm{e}-7$ & $5.91857 \mathrm{e}-9$ & $1.36581 \mathrm{e}-9$ \\
0.7 & 0.81923192051904 & $1.05020 \mathrm{e}-5$ & $6.25059 \mathrm{e}-7$ & $1.43766 \mathrm{e}-7$ & $9.14354 \mathrm{e}-9$ & $1.45891 \mathrm{e}-9$ \\
0.8 & 0.78086880944303 & $1.36172 \mathrm{e}-5$ & $1.04964 \mathrm{e}-6$ & $1.59582 \mathrm{e}-7$ & $1.10759 \mathrm{e}-8$ & $1.41417 \mathrm{e}-9$ \\
0.9 & 0.743294146247166 & $1.60925 \mathrm{e}-5$ & $1.51021 \mathrm{e}-6$ & $1.60847 \mathrm{e}-7$ & $1.20134 \mathrm{e}-8$ & $1.26230 \mathrm{e}-9$ \\
\hline
\end{tabular}

subject to the boundary conditions

$$
\mu(0)=1, \quad \mu^{\prime}(0)=0 .
$$

The exact solution is $\mu(x)=\frac{1}{\sqrt{1+x^{2}}}$. We solve this equation by the proposed method with $k=2,3$ and $M=3,4,5,6,7$. Tables 5 and 6 show the comparison between the absolute error of exact and approximate solutions for various values of $M$ with $k=2,3$.

Example 4 Consider the following nonlinear Lane-Emden equation [20]:

$$
\mu^{\prime \prime}(x)+\frac{2}{x} \mu^{\prime}(x)+4\left(2 e^{\mu(x)}+e^{\frac{\mu(x)}{2}}\right)=0, \quad 0<x<1,
$$


Table 7 Comparison of absolute errors for Example $4(k=2)$

\begin{tabular}{lllllll}
\hline $\boldsymbol{x}$ & Exact solution & $\begin{array}{l}\text { Absolute error } \\
\mathbf{M = 3}\end{array}$ & $\begin{array}{l}\text { Absolute error } \\
\boldsymbol{M = 4}\end{array}$ & $\begin{array}{l}\text { Absolute error } \\
\boldsymbol{M = 5}\end{array}$ & $\begin{array}{l}\text { Absolute error } \\
\boldsymbol{M = 6}\end{array}$ & $\begin{array}{l}\text { Absolute error } \\
\boldsymbol{M = ~}\end{array}$ \\
\hline 0.1 & -0.0199006617063362 & $7.12293 \mathrm{e}-5$ & $4.60723 \mathrm{e}-6$ & $1.03881 \mathrm{e}-6$ & $1.97866 \mathrm{e}-9$ & $9.02739 \mathrm{e}-9$ \\
0.2 & -0.0784414263065627 & $1.66987 \mathrm{e}-6$ & $6.19572 \mathrm{e}-7$ & $6.99131 \mathrm{e}-7$ & $1.51682 \mathrm{e}-8$ & $1.68836 \mathrm{e}-9$ \\
0.3 & -0.172355392482105 & $1.21751 \mathrm{e}-4$ & $1.76275 \mathrm{e}-6$ & $3.42269 \mathrm{e}-7$ & $2.01945 \mathrm{e}-8$ & $6.31737 \mathrm{e}-9$ \\
0.4 & -0.296840010236547 & $2.74878 \mathrm{e}-5$ & $1.49126 \mathrm{e}-6$ & $1.06726 \mathrm{e}-6$ & $5.07048 \mathrm{e}-9$ & $9.34269 \mathrm{e}-9$ \\
0.5 & -0.44628710262842 & $1.06032 \mathrm{e}-5$ & $2.81301 \mathrm{e}-6$ & $4.23783 \mathrm{e}-8$ & $4.20504 \mathrm{e}-8$ & $1.76553 \mathrm{e}-10$ \\
0.6 & -0.614969399495921 & $2.72115 \mathrm{e}-4$ & $1.79133 \mathrm{e}-5$ & $8.97718 \mathrm{e}-6$ & $5.75857 \mathrm{e}-7$ & $1.78242 \mathrm{e}-7$ \\
0.7 & -0.797552239914736 & $4.58685 \mathrm{e}-4$ & $2.48304 \mathrm{e}-5$ & $1.45806 \mathrm{e}-5$ & $9.99011 \mathrm{e}-7$ & $2.95038 \mathrm{e}-7$ \\
0.8 & -0.989392483672214 & $5.79446 \mathrm{e}-4$ & $2.95584 \mathrm{e}-5$ & $1.83900 \mathrm{e}-5$ & $1.27201 \mathrm{e}-6$ & $3.69959 \mathrm{e}-7$ \\
$0.9-1.18665369055547$ & $6.57413 \mathrm{e}-4$ & $3.25099 \mathrm{e}-5$ & $2.04445 \mathrm{e}-5$ & $1.43231 \mathrm{e}-6$ & $4.14540 \mathrm{e}-7$ \\
\hline
\end{tabular}

Table 8 Comparison of absolute errors for Example $4(k=3)$

\begin{tabular}{lllllll}
\hline $\boldsymbol{x}$ & Exact solution & $\begin{array}{l}\text { Absolute error } \\
\boldsymbol{M = 3}\end{array}$ & $\begin{array}{l}\text { Absolute error } \\
\mathbf{M = 4}\end{array}$ & $\begin{array}{l}\text { Absolute error } \\
\mathbf{M = 5}\end{array}$ & $\begin{array}{l}\text { Absolute error } \\
\boldsymbol{M = 6}\end{array}$ & $\begin{array}{l}\text { Absolute error } \\
\boldsymbol{M}=\mathbf{7}\end{array}$ \\
\hline 0.1 & -0.0199006617063362 & $1.64143 \mathrm{e}-8$ & $7.15543 \mathrm{e}-8$ & $4.63072 \mathrm{e}-9$ & $1.09733 \mathrm{e}-10$ & $4.51913 \mathrm{e}-12$ \\
0.2 & -0.0784414263065627 & $4.26643 \mathrm{e}-7$ & $7.99963 \mathrm{e}-8$ & $7.54245 \mathrm{e}-9$ & $8.38618 \mathrm{e}-11$ & $2.46411 \mathrm{e}-11$ \\
$0.3-0.172355392482105$ & $1.45496 \mathrm{e}-6$ & $1.90341 \mathrm{e}-7$ & $3.52023 \mathrm{e}-8$ & $4.66119 \mathrm{e}-10$ & $5.31878 \mathrm{e}-10$ \\
$0.4-0.296840010236547$ & $7.54378 \mathrm{e}-6$ & $7.77384 \mathrm{e}-7$ & $8.52194 \mathrm{e}-8$ & $1.55276 \mathrm{e}-10$ & $1.16498 \mathrm{e}-9$ \\
0.5 & -0.44628710262842 & $7.18512 \mathrm{e}-6$ & $1.12532 \mathrm{e}-6$ & $1.07965 \mathrm{e}-7$ & $6.28860 \mathrm{e}-10$ & $1.47615 \mathrm{e}-9$ \\
$0.6-0.614969399495921$ & $1.65622 \mathrm{e}-5$ & $1.03010 \mathrm{e}-7$ & $1.82404 \mathrm{e}-7$ & $7.06173 \mathrm{e}-9$ & $1.72201 \mathrm{e}-9$ \\
$0.7-0.797552239914736$ & $2.32175 \mathrm{e}-5$ & $6.06844 \mathrm{e}-7$ & $2.25375 \mathrm{e}-7$ & $1.22023 \mathrm{e}-8$ & $1.80556 \mathrm{e}-9$ \\
$0.8-0.989392483672214$ & $2.86341 \mathrm{e}-5$ & $1.40638 \mathrm{e}-6$ & $2.44832 \mathrm{e}-7$ & $1.51659 \mathrm{e}-8$ & $1.67463 \mathrm{e}-9$ \\
$0.9-1.18665369055547$ & $3.30731 \mathrm{e}-5$ & $2.31416 \mathrm{e}-6$ & $2.41118 \mathrm{e}-7$ & $1.67655 \mathrm{e}-8$ & $1.39453 \mathrm{e}-9$ \\
\hline
\end{tabular}

subject to the boundary conditions

$$
\mu(0)=0, \quad \mu^{\prime}(0)=0 .
$$

The exact solution is $\mu(x)=-2 \ln \left(1+x^{2}\right)$. We solve this equation by the proposed method with $k=2,3$ and $M=3,4,5,6,7$. Tables 7 and 8 show the comparison between the absolute error of exact and approximate solutions for various values of $M$ with $k=2,3$.

Example 5 Consider the following linear Lane-Emden equation:

$$
\mu^{\prime \prime}(x)+\frac{1}{x} \mu^{\prime}(x)+\mu(x)=x^{2}-x^{3}-9 x+4, \quad 0<x<1,
$$

subject to the boundary conditions

$$
\mu^{\prime}(0)=0, \quad \mu(1)=0 .
$$

The exact solution is $\mu(x)=x^{2}-x^{3}$. Next, we will give the approximate solution for this equation by the proposed method with $k=2$ and $M=3$. In this case, we have a linear system of six equations. By solving this system, we obtain

$$
\begin{aligned}
& c_{1,0}=-0.500000007308766, \quad c_{1,1}=0.75000001267439, \\
& c_{1,2}=-5.65363067472902 e-9, \\
& c_{2,0}=-2.00000002273819, \quad c_{2,1}=0.749999998967796, \\
& c_{1,2}=1.29351160060853 e-8 .
\end{aligned}
$$


Table 9 Comparison of absolute errors for Example $5(k=2,3)$

\begin{tabular}{llllll}
\hline $\boldsymbol{x}$ & Exact solution & $\begin{array}{l}\text { Absolute error } \\
\boldsymbol{k}=\mathbf{2} \boldsymbol{M} \mathbf{M = 3}\end{array}$ & $\begin{array}{l}\text { Absolute error } \\
\boldsymbol{k}=\mathbf{2} \boldsymbol{\mathbf { M } = \mathbf { 4 }}\end{array}$ & $\begin{array}{l}\text { Absolute error } \\
\boldsymbol{k}=\mathbf{3}, \boldsymbol{M}=\mathbf{3}\end{array}$ & $\begin{array}{l}\text { Absolute error } \\
\boldsymbol{k}=\mathbf{3}, \boldsymbol{M}=\mathbf{4}\end{array}$ \\
\hline 0.1 & 0.009 & $8.96289 \mathrm{e}-10$ & $3.92161 \mathrm{e}-10$ & $5.95982 \mathrm{e}-12$ & $7.96377 \mathrm{e}-12$ \\
0.2 & 0.032 & $8.72558 \mathrm{e}-10$ & $3.32835 \mathrm{e}-10$ & $2.50069 \mathrm{e}-11$ & $1.72426 \mathrm{e}-11$ \\
0.3 & 0.063 & $8.44767 \mathrm{e}-10$ & $3.09044 \mathrm{e}-10$ & $3.99208 \mathrm{e}-11$ & $2.53607 \mathrm{e}-11$ \\
0.4 & 0.096 & $8.01978 \mathrm{e}-10$ & $3.03655 \mathrm{e}-10$ & $6.62202 \mathrm{e}-11$ & $1.77049 \mathrm{e}-11$ \\
0.5 & 0.125 & $7.15163 \mathrm{e}-10$ & $2.80179 \mathrm{e}-10$ & $9.72653 \mathrm{e}-11$ & $1.43694 \mathrm{e}-11$ \\
0.6 & 0.144 & $7.48198 \mathrm{e}-10$ & $3.93943 \mathrm{e}-12$ & $9.33972 \mathrm{e}-11$ & $2.52972 \mathrm{e}-11$ \\
0.7 & 0.147 & $9.12601 \mathrm{e}-10$ & $1.80271 \mathrm{e}-10$ & $8.55649 \mathrm{e}-11$ & $4.49204 \mathrm{e}-11$ \\
0.8 & 0.128 & $9.68271 \mathrm{e}-10$ & $6.99664 \mathrm{e}-11$ & $1.43124 \mathrm{e}-10$ & $4.37169 \mathrm{e}-11$ \\
0.9 & 0.081 & $7.16510 \mathrm{e}-10$ & $3.93030 \mathrm{e}-11$ & $3.19812 \mathrm{e}-10$ & $4.91018 \mathrm{e}-11$ \\
\hline
\end{tabular}

Table 10 Comparison of absolute errors for Example $6(k=2)$

\begin{tabular}{lllllll}
\hline $\boldsymbol{x}$ & Exact solution & $\begin{array}{l}\text { Absolute error } \\
\mathbf{M = 3}\end{array}$ & $\begin{array}{l}\text { Absolute error } \\
\mathbf{M = 4}\end{array}$ & $\begin{array}{l}\text { Absolute error } \\
\boldsymbol{M = 5}\end{array}$ & $\begin{array}{l}\text { Absolute error } \\
\boldsymbol{M = 6}\end{array}$ & $\begin{array}{l}\text { Absolute error } \\
\boldsymbol{M}=\mathbf{7}\end{array}$ \\
\hline 0.1 & 0.313265850498063 & $2.55607 \mathrm{e}-7$ & $8.14189 \mathrm{e}-7$ & $1.81121 \mathrm{e}-8$ & $1.21233 \mathrm{e}-9$ & $3.14035 \mathrm{e}-10$ \\
0.2 & 0.3030154228323 & $4.26548 \mathrm{e}-7$ & $8.60558 \mathrm{e}-7$ & $1.57268 \mathrm{e}-8$ & $1.29968 \mathrm{e}-9$ & $3.06900 \mathrm{e}-10$ \\
0.3 & 0.286047265304854 & $1.31980 \mathrm{e}-6$ & $8.60956 \mathrm{e}-7$ & $1.57118 \mathrm{e}-8$ & $1.31094 \mathrm{e}-9$ & $2.98647 \mathrm{e}-10$ \\
0.4 & 0.262531127456033 & $8.54592 \mathrm{e}-7$ & $8.45643 \mathrm{e}-7$ & $1.38501 \mathrm{e}-8$ & $1.26470 \mathrm{e}-9$ & $2.88410 \mathrm{e}-10$ \\
0.5 & 0.232696783873834 & $5.95794 \mathrm{e}-7$ & $8.74915 \mathrm{e}-7$ & $1.51067 \mathrm{e}-8$ & $1.33441 \mathrm{e}-9$ & $2.82368 \mathrm{e}-10$ \\
0.6 & 0.196826805692954 & $4.48716 \mathrm{e}-8$ & $6.66434 \mathrm{e}-7$ & $1.27714 \mathrm{e}-8$ & $1.07644 \mathrm{e}-9$ & $2.13563 \mathrm{e}-10$ \\
0.7 & 0.155248106682756 & $2.34595 \mathrm{e}-7$ & $4.72070 \mathrm{e}-7$ & $7.86159 \mathrm{e}-9$ & $7.56736 \mathrm{e}-10$ & $1.50629 \mathrm{e}-10$ \\
0.8 & 0.108322763444465 & $9.46220 \mathrm{e}-7$ & $3.02305 \mathrm{e}-7$ & $5.09872 \mathrm{e}-9$ & $4.93439 \mathrm{e}-10$ & $9.44874 \mathrm{e}-11$ \\
0.9 & 0.0564386024692362 & $4.47318 \mathrm{e}-7$ & $1.49726 \mathrm{e}-7$ & $1.05906 \mathrm{e}-9$ & $2.86694 \mathrm{e}-10$ & $4.39479 \mathrm{e}-11$ \\
\hline
\end{tabular}

So the approximate solution is given by

$$
\mu(x)=\left\{\begin{array}{c}
9.08805 \times 10^{-10}+1.00000 x^{2}-0.999999 x^{3} \\
\quad-7.53817 \times 10^{-9} x^{4}, \quad 0 \leq x<\frac{1}{2} \\
1.38213 \times 10^{-8}-6.80971 \times 10^{-8} x+1.00000 x^{2}-1.00000 x^{3} \\
\quad+1.72468 \times 10^{-8} x^{4}, \quad \frac{1}{2} \leq x \leq 1
\end{array}\right.
$$

Obviously, the Laguerre wavelets solution is very close to the exact solution. Table 9 shows the comparison between the absolute error of exact and approximate solutions for various values of $M$ with $k=2,3$.

Example 6 Consider the following nonlinear Lane-Emden equation $[5,16,21]$ :

$$
\mu^{\prime \prime}(x)+\frac{1}{x} \mu^{\prime}(x)+e^{\mu(x)}=0, \quad 0<x<1,
$$

subject to the boundary conditions

$$
\mu^{\prime}(0)=0, \quad \mu(1)=0 .
$$

The exact solution is $\mu(x)=2 \ln \left(\frac{4-2 \sqrt{2}}{(3-2 \sqrt{2}) x^{2}+1}\right)$. We solve this equation by the proposed method with $k=2,3$ and $M=3,4,5,6,7$. Tables 10 and 11 show the comparison between the absolute error of exact and approximate solutions for various values of $M$ with $k=2,3$. The numerical solutions obtained by orthonormal Bernoulli polynomial approach [16] is presented in Table 12. 
Table 11 Comparison of absolute errors for Example $6(k=3)$

\begin{tabular}{lllllll}
\hline $\boldsymbol{x}$ & Exact solution & $\begin{array}{l}\text { Absolute error } \\
\boldsymbol{M = 3}\end{array}$ & $\begin{array}{l}\text { Absolute error } \\
\boldsymbol{M = 4}\end{array}$ & $\begin{array}{l}\text { Absolute error } \\
\boldsymbol{M = 5}\end{array}$ & $\begin{array}{l}\text { Absolute error } \\
\boldsymbol{M = 6}\end{array}$ & $\begin{array}{l}\text { Absolute error } \\
\boldsymbol{M = ~}\end{array}$ \\
\hline 0.1 & 0.313265850498063 & $1.12155 \mathrm{e}-7$ & $7.47073 \mathrm{e}-8$ & $7.77016 \mathrm{e}-11$ & $2.25000 \mathrm{e}-11$ & $1.12567 \mathrm{e}-11$ \\
0.2 & 0.3030154228323 & $1.18254 \mathrm{e}-7$ & $7.42881 \mathrm{e}-8$ & $7.21018 \mathrm{e}-11$ & $2.28459 \mathrm{e}-11$ & $1.06202 \mathrm{e}-11$ \\
0.3 & 0.286047265304854 & $8.26092 \mathrm{e}-8$ & $6.72829 \mathrm{e}-8$ & $1.32635 \mathrm{e}-10$ & $1.64894 \mathrm{e}-11$ & $9.71828 \mathrm{e}-12$ \\
0.4 & 0.262531127456033 & $7.92919 \mathrm{e}-8$ & $5.59685 \mathrm{e}-8$ & $1.80870 \mathrm{e}-10$ & $5.77443 \mathrm{e}-12$ & $8.57608 \mathrm{e}-12$ \\
0.5 & 0.232696783873834 & $3.19442 \mathrm{e}-8$ & $4.68132 \mathrm{e}-8$ & $2.27523 \mathrm{e}-10$ & $2.24772 \mathrm{e}-12$ & $7.28159 \mathrm{e}-12$ \\
0.6 & 0.196826805692954 & $1.34284 \mathrm{e}-8$ & $3.36344 \mathrm{e}-8$ & $2.33153 \mathrm{e}-10$ & $8.22350 \mathrm{e}-12$ & $5.78329 \mathrm{e}-12$ \\
0.7 & 0.155248106682756 & $6.97215 \mathrm{e}-9$ & $2.21505 \mathrm{e}-8$ & $2.24769 \mathrm{e}-10$ & $1.30745 \mathrm{e}-11$ & $4.31280 \mathrm{e}-12$ \\
0.8 & 0.108322763444465 & $3.25668 \mathrm{e}-8$ & $1.34976 \mathrm{e}-8$ & $1.93600 \mathrm{e}-10$ & $1.16738 \mathrm{e}-11$ & $2.82023 \mathrm{e}-12$ \\
0.9 & 0.0564386024692362 & $8.78766 \mathrm{e}-9$ & $6.50385 \mathrm{e}-9$ & $8.87945 \mathrm{e}-11$ & $5.50390 \mathrm{e}-12$ & $1.33990 \mathrm{e}-12$ \\
\hline
\end{tabular}

Table 12 Absolute errors for Example 6 in [16]

\begin{tabular}{llll}
\hline $\boldsymbol{x}$ & Exact solution & $\mathbf{N = 1 0}$ & $\boldsymbol{N = 1 4}$ \\
\hline 0.1 & 0.313265850498063 & $1.05 \mathrm{e}-7$ & $6.69 \mathrm{e}-8$ \\
0.2 & 0.3030154228323 & $6.33 \mathrm{e}-9$ & $7.87 \mathrm{e}-9$ \\
0.3 & 0.286047265304854 & $5.91 \mathrm{e}-8$ & $6.92 \mathrm{e}-9$ \\
0.4 & 0.262531127456033 & $2.12 \mathrm{e}-7$ & $2.87 \mathrm{e}-8$ \\
0.5 & 0.232696783873834 & $1.00 \mathrm{e}-8$ & $7.40 \mathrm{e}-10$ \\
0.6 & 0.196826805692954 & $5.36 \mathrm{e}-7$ & $6.32 \mathrm{e}-8$ \\
0.7 & 0.155248106682756 & $4.25 \mathrm{e}-8$ & $6.95 \mathrm{e}-8$ \\
0.8 & 0.108322763444465 & $8.32 \mathrm{e}-7$ & $3.38 \mathrm{e}-9$ \\
0.9 & 0.0564386024692362 & $4.67 \mathrm{e}-8$ & $7.85 \mathrm{e}-8$ \\
\hline
\end{tabular}

Table 13 The numerical results of Example 7 with $k=3$

\begin{tabular}{llllll}
\hline $\boldsymbol{x}$ & $\boldsymbol{M = 3}$ & $\boldsymbol{M = 4}$ & $\boldsymbol{M = 5}$ & $\boldsymbol{M = 6}$ & $\begin{array}{l}\text { Method in [16] } \\
\text { with } \boldsymbol{n = 1 4}\end{array}$ \\
\hline 0.1 & 0.829706090093794 & 0.82970609213969 & 0.829706092330806 & 0.829706092433877 & 0.82970609243390 \\
0.2 & 0.833374731260013 & 0.8333747332988822 & 0.833374733492775 & 0.833374733591078 & 0.833374733559110 \\
0.3 & 0.839489911535405 & 0.839489913690883 & 0.8394899138631 & 0.83948991395376 & 0.83948991395381 \\
0.4 & 0.848052782678684 & 0.848052784769831 & 0.848052784915137 & 0.848052784996097 & 0.84805278499617 \\
0.5 & 0.85906492472985 & 0.859064926965802 & 0.859064927099445 & 0.85906492716925 & 0.85906492716933 \\
0.6 & 0.872528317441265 & 0.872528319803658 & 0.872528319900291 & 0.87252831995828 & 0.87252831995828 \\
0.7 & 0.888445303225948 & 0.888445305504435 & 0.888445305576983 & 0.888445305623171 & 0.88844530562329 \\
0.8 & 0.906818545614923 & 0.906818547978651 & 0.906818548031817 & 0.906818548066776 & 0.90681854806690 \\
0.9 & 0.927650986181403 & 0.927650988306455 & 0.927650988340659 & 0.927650988365551 & 0.92765098836568 \\
\hline
\end{tabular}

Example 7 Consider the oxygen diffusion problem

$$
\mu^{\prime \prime}(x)+\frac{2}{x} \mu^{\prime}(x)=\frac{0.76129 \mu(x)}{\mu(x)+0.03119}, \quad 0<x<1
$$

subject to the boundary conditions

$$
\mu^{\prime}(0)=0, \quad 5 \mu(1)+\mu^{\prime}(1)=5,
$$

where the exact solution is unknown. Now we solve this equation by the proposed method with $k=3$ and $M=3,4,5,6$. These results are in good agreement with that of [16] and the results are tabulated in Table 13. 
Table 14 Comparison of absolute errors for Example $8(k=2)$

\begin{tabular}{lllllll}
\hline $\boldsymbol{x}$ & Exact solution & $\begin{array}{l}\text { Absolute error } \\
\boldsymbol{M = 3}\end{array}$ & $\begin{array}{l}\text { Absolute error } \\
\mathbf{M = 4}\end{array}$ & $\begin{array}{l}\text { Absolute error } \\
\boldsymbol{M = 5}\end{array}$ & $\begin{array}{l}\text { Absolute error } \\
\boldsymbol{M = 6}\end{array}$ & $\begin{array}{l}\text { Absolute error } \\
\boldsymbol{M = ~}\end{array}$ \\
\hline 0.1 & 0.100665339016074 & $1.99905 \mathrm{e}-4$ & $2.28846 \mathrm{e}-5$ & $1.72044 \mathrm{e}-6$ & $4.80911 \mathrm{e}-7$ & $5.02872 \mathrm{e}-8$ \\
0.2 & 0.205291382243876 & $1.91458 \mathrm{e}-4$ & $2.34563 \mathrm{e}-5$ & $1.63517 \mathrm{e}-6$ & $4.79635 \mathrm{e}-7$ & $4.89559 \mathrm{e}-8$ \\
0.3 & 0.317687905980875 & $1.67910 \mathrm{e}-4$ & $2.28553 \mathrm{e}-5$ & $1.57308 \mathrm{e}-6$ & $4.65931 \mathrm{e}-7$ & $4.71142 \mathrm{e}-8$ \\
0.4 & 0.441387397450343 & $1.61539 \mathrm{e}-4$ & $2.17240 \mathrm{e}-5$ & $1.47290 \mathrm{e}-6$ & $4.39266 \mathrm{e}-7$ & $4.45112 \mathrm{e}-8$ \\
0.5 & 0.579559511251008 & $1.51975 \mathrm{e}-4$ & $2.13789 \mathrm{e}-5$ & $1.36356 \mathrm{e}-6$ & $4.18131 \mathrm{e}-7$ & $4.14292 \mathrm{e}-8$ \\
0.6 & 0.734970520367994 & $9.83797 \mathrm{e}-5$ & $1.29024 \mathrm{e}-5$ & $9.77157 \mathrm{e}-7$ & $2.95283 \mathrm{e}-7$ & $2.86342 \mathrm{e}-8$ \\
0.7 & 0.909981686939921 & $4.84761 \mathrm{e}-5$ & $5.13638 \mathrm{e}-6$ & $6.43969 \mathrm{e}-7$ & $1.71463 \mathrm{e}-7$ & $1.62533 \mathrm{e}-8$ \\
0.8 & 1.10657514524663 & $7.88980 \mathrm{e}-6$ & $1.98660 \mathrm{e}-6$ & $2.77744 \mathrm{e}-7$ & $5.15655 \mathrm{e}-8$ & $4.25484 \mathrm{e}-9$ \\
0.9 & 1.32639533423358 & $3.47573 \mathrm{e}-5$ & $7.91713 \mathrm{e}-6$ & $5.42440 \mathrm{e}-8$ & $5.87647 \mathrm{e}-8$ & $6.74598 \mathrm{e}-9$ \\
\hline
\end{tabular}

Table 15 Comparison of absolute errors for Example $8(k=3)$

\begin{tabular}{lllllll}
\hline $\boldsymbol{x}$ & Exact solution & $\begin{array}{l}\text { Absolute error } \\
\boldsymbol{M = 3}\end{array}$ & $\begin{array}{l}\text { Absolute error } \\
\boldsymbol{M = 4}\end{array}$ & $\begin{array}{l}\text { Absolute error } \\
\boldsymbol{M = 5}\end{array}$ & $\begin{array}{l}\text { Absolute error } \\
\boldsymbol{M = 6}\end{array}$ & $\begin{array}{l}\text { Absolute error } \\
\boldsymbol{M =}=\mathbf{7}\end{array}$ \\
\hline 0.1 & 0.100665339016074 & $2.00943 \mathrm{e}-5$ & $2.49095 \mathrm{e}-6$ & $7.39087 \mathrm{e}-8$ & $8.51470 \mathrm{e}-9$ & $9.01484 \mathrm{e}-12$ \\
0.2 & 0.205291382243876 & $1.91219 \mathrm{e}-5$ & $2.43795 \mathrm{e}-6$ & $7.17544 \mathrm{e}-8$ & $8.33840 \mathrm{e}-9$ & $3.18067 \mathrm{e}-12$ \\
0.3 & 0.317687905980875 & $1.75163 \mathrm{e}-5$ & $2.21871 \mathrm{e}-6$ & $6.28094 \mathrm{e}-8$ & $7.40476 \mathrm{e}-9$ & $3.37537 \mathrm{e}-11$ \\
0.4 & 0.441387397450343 & $1.46803 \mathrm{e}-5$ & $1.89551 \mathrm{e}-6$ & $4.88112 \mathrm{e}-8$ & $5.95511 \mathrm{e}-9$ & $9.98910 \mathrm{e}-11$ \\
0.5 & 0.579559511251008 & $1.21171 \mathrm{e}-5$ & $1.59113 \mathrm{e}-6$ & $3.61323 \mathrm{e}-8$ & $4.63996 \mathrm{e}-9$ & $1.48223 \mathrm{e}-10$ \\
0.6 & 0.734970520367994 & $8.42905 \mathrm{e}-6$ & $1.08465 \mathrm{e}-6$ & $2.44077 \mathrm{e}-8$ & $3.18752 \mathrm{e}-9$ & $1.28915 \mathrm{e}-10$ \\
0.7 & 0.909981686939921 & $5.01234 \mathrm{e}-6$ & $5.98191 \mathrm{e}-7$ & $1.33754 \mathrm{e}-8$ & $1.77942 \mathrm{e}-9$ & $1.03023 \mathrm{e}-10$ \\
0.8 & 1.10657514524663 & $1.47814 \mathrm{e}-6$ & $1.20797 \mathrm{e}-7$ & $4.04928 \mathrm{e}-9$ & $6.02002 \mathrm{e}-10$ & $6.26907 \mathrm{e}-11$ \\
0.9 & 1.32639533423358 & $1.54737 \mathrm{e}-6$ & $3.27982 \mathrm{e}-7$ & $2.46634 \mathrm{e}-9$ & $3.25944 \mathrm{e}-10$ & $2.24953 \mathrm{e}-12$ \\
\hline
\end{tabular}

Example 8 Consider the following nonlinear singular two-point BVP:

$$
\mu^{\prime \prime}(x)+\frac{1}{x} \mu^{\prime}(x)+\frac{\mu^{2}(x)}{x(1-x)}=4 \arctan x+\frac{1+3 x^{2}}{x\left(1+x^{2}\right)}+\frac{\left(1+x^{2}\right)^{2} \arctan ^{2} x}{x(1-x)}, \quad 0<x<1,
$$

subject to the boundary conditions

$$
\mu(0)+\mu^{\prime}(0)=1, \quad \mu(1)+\mu^{\prime}(1)=4.14159265358979 .
$$

The exact solution is $\mu(x)=\left(1+x^{2}\right) \arctan x$. We solve this equation by the proposed method with $k=2,3$ and $M=3,4,5,6,7$. Tables 14 and 15 show the comparison between the absolute error of exact and approximate solutions for various values of $M$ with $k=2,3$.

\section{Conclusion}

The main goal of this paper is to develop an efficient and accurate method to solve linear or nonlinear singular boundary value problems with four different types' initial boundary conditions and mixed boundary conditions. The Laguerre wavelets operational matrix of integration together with the collocation method is utilized to reduce the problem to the solution of linear or nonlinear algebraic equations. One of the main advantages of the developed algorithm is that it does not require any modification while switching from the linear case to the nonlinear case. Another one is that high accuracy approximate solutions are achieved using very small values of $k$ and $M$. Illustrative examples are included to demonstrate the validity and applicability of the proposed method. 
Authors' contributions

FZ and XX came up with the idea of this paper. FZ completed the proofs of the results, and XX designed a MATLAB program to simulate the results of examples. FZ and XX wrote the manuscript. All authors read and approved the final manuscript.

\section{Acknowledgements}

This work is supported by the Education Department Youth Science Foundation of Jiangxi Province (Grant No. GJJ14492), the Youth Science Foundation of Jiangxi Province (Grant No. 20151BAB211004), and the Ph.D. Research Startup Foundation of East China University of Technology (Grant No. DHBK2012205).

\section{Received: 16 August 2015 Accepted: 11 January 2016 Published online: 25 January 2016}

\section{References}

1. Kumar, $\mathrm{M}$, Singh, $\mathrm{N}$ : A collection of computational techniques for solving singular boundary-value problems. Adv. Eng. Softw. 40, 288-297 (2009)

2. Doha, EH, Abd-Elhameed, WM, Youssri, YH: Second kind Chebyshev operational matrix algorithm for solving differential equations of Lane-Emden type. New Astron. 23/24, 113-117 (2013)

3. Kanth, ASVR, Aruna, K: He's variational iteration method for treating nonlinear singular boundary value problems. Comput. Math. Appl. 60, 821-829 (2010)

4. Chang, SH: Taylor series method for solving a class of nonlinear singular boundary value problems arising in applied science. Appl. Math. Comput. 235, 110-117 (2014)

5. Singh, R, Kumar, J: An efficient numerical technique for the solution of nonlinear singular boundary value problems. Comput. Phys. Commun. 185, 1282-1289 (2014)

6. Sahlan, MN, Hashemizadeh, E: Wavelet Galerkin method for solving nonlinear singular boundary value problems arising in physiology. Appl. Math. Comput. 250, 260-269 (2015)

7. Arqub, OA, Abo-Hammour, Z, Momani, S, Shawagfeh, N: Solving singular two-point boundary value problems using continuous genetic algorithm. Abstr. Appl. Anal. 2012, Article ID 205391 (2012)

8. Ebaid, A: A new analytical and numerical treatment for singular two-point boundary value problems via the Adomian decomposition method. J. Comput. Appl. Math. 235, 1914-1924 (2011)

9. Goh, J, Majid, AA, Ismail, AIM: A quartic B-spline for second-order singular boundary value problems. Comput. Math. Appl. 64, 115-120 (2012)

10. Nasab, AK, Kılıçman, A, Atabakan, ZP, Leong, WJ: A numerical approach for solving nonlinear Lane-Emden type equations arising in astrophysics. New Astron. 34, 178-186 (2015)

11. Pandey, RK, Kumar, N, Bhardwaj, A, Dutta, G: Solution of Lane-Emden type equations using Legendre operational matrix of differentiation. Appl. Math. Comput. 218, 7629-7637 (2012)

12. Pandey, RK, Kumar, N: Solution of Lane-Emden type equations using Bernstein operational matrix of differentiation. New Astron. 17, 303-308 (2012)

13. Gürbüz, B, Sezer, M: Laguerre polynomial approach for solving Lane-Emden type functional differential equations. Appl. Math. Comput. 242, 255-264 (2014)

14. Parand, K, Nikarya, M, Rad, JA: Solving non-linear Lane-Emden type equations using Bessel orthogonal functions collocation method. Celest. Mech. Dyn. Astron. 116, 97-107 (2013)

15. Öztürk, Y, Gülsu, M: An approximation algorithm for the solution of the Lane-Emden type equations arising in astrophysics and engineering using Hermite polynomials. Comput. Appl. Math. 33, 131-145 (2014)

16. Mohsenyzadeh, M, Maleknejad, K, Ezzati, R: A numerical approach for the solution of a class of singular boundary value problems arising in physiology. Adv. Differ. Equ. 2015, Article ID 231 (2015)

17. Yousefi, SA: Legendre wavelets method for solving differential equations of Lane-Emden type. Appl. Math. Comput. $181,1417-1422(2006)$

18. Nasab, AK, Kılıçman, A, Babolian, E, Atabakan, ZP: Wavelet analysis method for solving linear and nonlinear singular boundary value problems. Appl. Math. Model. 37, 5876-5886 (2013)

19. Kaur, H, Mittal, RC, Mishra, V: Haar wavelet approximate solutions for the generalized Lane-Emden equations arising in astrophysics. Comput. Phys. Commun. 184, 2169-2177 (2013)

20. Mehrpouya, MA: An efficient pseudospectral method for numerical solution of nonlinear singular initial and boundary value problems arising in astrophysics. Math. Methods Appl. Sci. (2015). doi:10.1002/mma.3763

21. Khuri, SA, Sayfy, A: A novel approach for the solution of a class of singular boundary value problems arising in physiology. Math. Comput. Model. 52, 626-636 (2010) 